\title{
MÉTODO COLORIMÉTRICO PARA DETERMINAÇÃO DE DIÓXIDO DE NITROGÊNIO ATMOSFÉRICO COM PRECONCENTRAÇÃO EM COLUNA DE C-18
}

\author{
Cássia Ugucione, José de Anchieta Gomes Neto e Arnaldo Alves Cardoso*
}

Departamento de Química Analítica, Instituto de Química de Araraquara, Universidade Estadual Paulista "Júlio de Mesquita Filho", CP 355, 14800-900 Araraquara - SP

Recebido em 7/12/00; aceito em 9/1/02

\begin{abstract}
COLORIMETRIC DETERMINATION OF ATMOSPHERIC NITROGEN DIOXIDE USING PRECONCENTRATION ON C-18 CARTRIDGE. An alternative analytical method for nitrogen dioxide $\left(\mathrm{NO}_{2}\right)$ in atmosphere was developed. The collection of $\mathrm{NO}_{2}$ is performed by a Sep-Pack C-18 cartridge impregnated with $11 \%(\mathrm{v} / \mathrm{v})$ of triethanolamine plus 3,6 \% (v/v) of ethylene glycol plus $25 \%(\mathrm{v} / \mathrm{v})$ of acetone combined solution. When the impregnating is used, $\mathrm{NO}_{2}$ is collected with good repeatibility $(\mathrm{CV}=$ $3,3 \%)$. The $\mathrm{NO}_{2}$ absorbed in the sampler was stripped from the sorbent with a methanol $5 \%(\mathrm{v} / \mathrm{v})$ aqueous solution and was determined by colorimetry as nitrite by using the Griess-Saltzman reagent. The detection limit of 1,4 ppb for 60 min sampling at $0,5 \mathrm{~L} \mathrm{~min}^{-1}$ flow rate was obtained. Preparation and conditioning procedures for TEA-C-18 cartridge, sampling flow rate, absorption capacity and interference of other species are discussed.
\end{abstract}

Keywords: nitrogen dioxide; triethanolamine; Griess Saltzman reagent.

\section{INTRODUÇÃO}

O gás nitrogênio é o principal componente em proporção do ar atmosférico. Além dele, várias outras espécies químicas que pertencem ao ciclo do nitrogênio são encontradas na forma de gás na atmosfera, as principais são os óxidos de nitrogênio e a amônia ${ }^{1,2}$. Atividades humanas, principalmente as relacionadas com indústrias e agricultura tem aumentado o aporte de compostos de nitrogênio para a biosfera criando distúrbios no ciclo natural do nitrogênio. Nos aglomerados urbanos o dióxido de nitrogênio $\left(\mathrm{NO}_{2}\right)$ é reconhecido como um dos principais poluentes da troposfera ${ }^{1,2}$. As reações entre $\mathrm{NO}_{2} \mathrm{e}$ outros compostos presentes na atmosfera são inúmeras e têm se mostrado cada vez mais complexas. $\mathrm{O} \mathrm{NO}_{2}$ está envolvido na produção e regulação de ozônio $\left(\mathrm{O}_{3}\right)$, na formação do "smog" fotoquímico, na produção de ácidos nitroso $\left(\mathrm{HNO}_{2}\right)$ e nítrico $\left(\mathrm{HNO}_{3}\right)$, trióxido de nitrogênio $\left(\mathrm{NO}_{3}\right)$, pentóxido de dinitrogênio $\left(\mathrm{N}_{2} \mathrm{O}_{5}\right)$, nitrato de peroxiacetila (PAN) e outros compostos orgânicos nitrados, além de ser um dos principais contribuintes para formação de chuvas ácidas ${ }^{1,2}$.

A emissão direta de $\mathrm{NO}_{2}$ para atmosfera é relativamente pequena. A rápida oxidação do óxido nítrico $(\mathrm{NO})$ que ocorre na atmosfera constitui-se na principal fonte de $\mathrm{NO}_{2}$ atmosférico. Atividades de microorganismos no solo são importantes fontes de emissão natural de NO. Porém, o excesso do $\mathrm{NO}_{2}$ responsável pelos problemas de poluição em centros urbanos tem como principal fonte, o NO resultante da queima de toda espécie de combustíveis pelas fontes móveis e estacionárias $^{3,4}$. Dados de 1978 para o Rio de Janeiro mostram que a emissão total de óxidos de nitrogênio foi de 63000 ton ano ${ }^{-1}$, com $92 \%$ da contribuição das fontes móveis. Já para a cidade de São Paulo em 1988, a emissão foi 245000 ton ano $^{-1}$, com $82 \%$ da contribuição das fontes móveis ${ }^{5}$. Como o gás $\mathrm{NO}_{2}$ está envolvido em muitas reações fotoquímicas, sua concentração na atmosfera, além de depender de fontes de emissão varia ao longo do dia e sazonalmente acompanhando as mudanças da intensidade da luz solar. Em ambientes fecha-

\footnotetext{
* e-mail: acardoso@iq.unesp.br
}

dos e pequenos, como cozinhas e interiores de veículos, a concentração pode ficar próxima de $200 \mathrm{ppb}^{6-8}$, com registros de níveis de até 1,5 ppm para ambientes providos de aquecedores ${ }^{9}$.

Estudos toxicológicos apontam o $\mathrm{NO}_{2}$ como o mais tóxico dentre os óxidos de nitrogênio. No pulmão pode formar nitrosoaminas, os quais são compostos com potencial ação cancerígena. Exposição a concentrações acima de $5 \mathrm{ppm}$, o $\mathrm{NO}_{2}$ pode aumentar a susceptibilidade de agentes bronquioconstritores e infecções respiratórias por bactérias, principalmente em crianças ${ }^{10}$.

Ar poluído com óxidos de nitrogênio pode afetar indiretamente a vegetação, já que o $\mathrm{NO}_{2}$ é um precursor do ozônio e este é um reconhecido composto fitotóxico. A deposição de compostos de nitrogênio no solo e na água pode afetar a sua acidez ou a sua composição natural. Um efeito do aumento da acidez do solo é o conseqüente aumento da biodisponibilidade do íon alumínio que resulta em danos para as raízes. A composição natural do solo é um fator importante para manter a biodiversidade vegetal. $\mathrm{O}$ nitrogênio é um nutriente limitante do crescimento de plantas em muitos ecossistemas. Muitas espécies de plantas estão adaptadas a condições de solo pobres em nitrogênio, e uma condição de enriquecimento de nitrogênio pode levar a mudanças na cobertura de vegetais de uma região afetando assim a biodiversidade das espécies locais ${ }^{6}$.

A demanda por monitoramento de espécies químicas de importância ambiental, como o $\mathrm{NO}_{2}$, é uma prática que tende a aumentar como conseqüência de avaliações ambientais necessárias para conhecimento e controle da poluição atmosférica sobre cidades e regiões próximas. Paralelamente estudos para reconhecer o grau de exposição ocupacional de trabalhadores em ambientes fechados são cada vez mais freqüentes. Entretanto, ampla aplicação de monitoramentos ambientais depende de métodos de análises químicas que sejam fáceis de usar, economicamente viáveis e se possível, que a determinação do sinal analítico utilize equipamento de baixo custo ou comum a qualquer laboratório de análise. O método desenvolvido por Saltzman ${ }^{11}$ em 1954 para $\mathrm{NO}_{2}$ é ainda recomendado em manuais recentes de amostragem e análise de $\operatorname{ar}^{12}$ e apresenta as características acima mencionadas. Neste procedimento, a amostragem é feita com o uso de borbulhadores e a determinação via formação de 
corante vermelho violeta (reação de Griess-Saltzman). Como a reação tem boa seletividade, sensibilidade e cinética relativamente rápida (15 min), o inconveniente maior recai para a etapa de amostragem, que utiliza um borbulhador de vidro que é pouco prático para ser usado em análises de campo. Para substituir o borbulhador de vidro como método de coleta do $\mathrm{NO}_{2}$, tem se buscado utilizar tubos coletores contendo sólidos com superfícies ativas para reter seletivamente o $\mathrm{NO}_{2}$ contido em amostras de ar. Diferentes materiais ${ }^{13-16}$, como a sílica-gel, peneira molecular, alumina ativada e dióxido de manganês $\left(\mathrm{MnO}_{2}\right)$ têm sido usados para coletar $\mathrm{NO}_{2}$. Porém a umidade é um inconveniente, já que pode gerar $\mathrm{NO}^{14}$. Para contornar este problema e melhorar a eficiência de coleta, as superfícies têm recebido um recobrimento de reagentes seletivos para o $\mathrm{NO}_{2}$, como a p-anisaldina ${ }^{17}$ e, com maior frequiência a trietanolamina (TEA) ${ }^{18-23}$.

Esse trabalho descreve um método para preconcentração de $\mathrm{NO}_{2}$ utilizando uma coluna de sílica gel imobilizada com grupos octadecil (C-18) impregnada com solução de TEA. O preparo e a utilização da coluna é relativamente simples, de fácil manipulação e ela pode ser reutilizada em diversas amostragens se convenientemente tratada. Após a extração do analito com solução de metanol, o $\mathrm{NO}_{2}$ é determinado por método colorimétrico utilizando a reação de GriessSaltzman.

\section{PARTE EXPERIMENTAL}

\section{Materiais}

As medidas de absorbância foram feitas com o espectrofotômetro HITACHI U-2000, com cubetas de vidro de $10 \mathrm{~mm}$. Como leito sorvente utilizou-se coluna C-18 (Sep-Pack cartridges Waters/ Millipore). Todos os reagentes utilizados foram de grau analítico, e água desionizada foi usada no preparo das soluções. O reagente de Griess-Saltzman foi preparado dissolvendo-se 5,0 g de ácido sulfanílico em solução contendo $600 \mathrm{~mL}$ de água, $140 \mathrm{~mL}$ de ácido acético glacial, $20 \mathrm{mg}$ de $\mathrm{N}$-(1-naftil)-etilenodiamina e diluindo-se para $1 \mathrm{~L}$ com água. A solução absorvente foi preparada dissolvendose $11 \mathrm{~mL}$ TEA, 3,6 mL de etilenoglicol e diluindo-se com $25 \mathrm{~mL}$ de acetona e completando se o volume até $100 \mathrm{~mL}$ com água.

\section{Condicionamento e preparo da coluna de C-18}

Lava-se o interior da coluna C-18 com $5 \mathrm{~mL}$ de solução $5 \%$ (v/v) de metanol. Em seguida, o excesso de solução de metanol é retirado passando-se pela coluna um fluxo de ar limpo. Finalmente, um volume de $5 \mathrm{~mL}$ da solução absorvente de TEA é introduzido vagarosamente pela coluna $\mathrm{C}-18$ e o excesso drenado com ar limpo.

\section{Geração de padrões gasosos de $\mathrm{NO}_{2}$}

Para avaliação do método proposto foi utilizado ar contendo quantidade conhecida de $\mathrm{NO}_{2}$. $\mathrm{O}$ esquema do sistema utilizado na geração do padrão gasoso de $\mathrm{NO}_{2}$ está ilustrado na Figura 1 . O ar ambiente foi previamente purificado para ser utilizado como diluente do $\mathrm{NO}_{2}$ emitido pelo tubo de permeação e gerar o padrão gasoso. A purificação do ar foi obtida, fazendo-o atravessar colunas de sílica gel e carvão ativo, respectivamente. Na seqüência, o ar purificado foi dividido em duas frações. Uma parte do ar limpo passa pela câmara de vidro contendo um tubo de permeação de $\mathrm{NO}_{2}$ (DYNACAL com taxa de permeação de $93,2 \mathrm{ng} \mathrm{NO} \min ^{-1} \pm 5 \%$ a $\left.30{ }^{\circ} \mathrm{C}\right)$. A fração restante é utilizada para diluir convenientemente o padrão gasoso e obter diferentes concentrações de $\mathrm{NO}_{2}$. A parte do padrão gasoso não utilizada foi descartada por uma linha de saída colocada anteriormente ao amostrador.

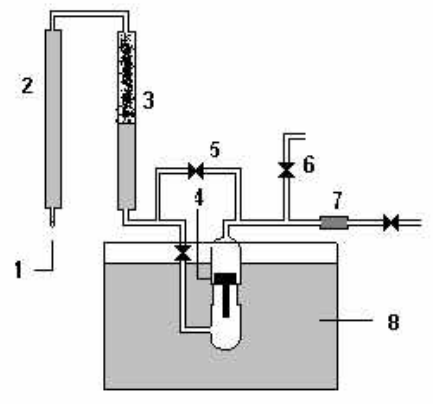

Figura 1. Sistema de geração de atmosfera padrão com diferentes concentrações de $\mathrm{NO}_{2}$. 1- entrada de ar comprimido,2 e 3-colunas de purificação de ar, 4- tubo de permeação de $\mathrm{NO}_{2}, 5$ - saída de ar para diluição, 6- saída de descarte, 7-tubo de amostragem, 8-banho termostatizado

\section{Protocolo de análise de $\mathrm{NO}_{2}$ presente nos padrões gasosos}

Para a coleta das misturas testes de $\mathrm{NO}_{2}$, conectou-se a coluna de C-18 condicionada com trietanolamina na linha de fluxo, como indicado na figura 1 . O padrão gasoso passa pela coluna de C-18 por um tempo preestabelecido, a maioria dos experimentos de amostragem foram feitos ao longo de 1 hora. Decorrido esse tempo, a coluna de C-18 é desconectada e guardada dentro de um saco plástico limpo para evitar contaminação. O material retido na coluna de C-18 é extraído passando se vagarosamente $5 \mathrm{~mL}$ de solução $5 \%$ (v/v) de metanol pelo interior da mesma com o auxílio de uma seringa comum. A solução eluída é recolhida em balão volumétrico de 10 $\mathrm{mL}$ e o volume completado com o reagente de Griess-Saltzman. Espera-se cerca de 15 min para que se complete a reação e faz-se a medida de absorbância $(\lambda=540 \mathrm{~nm})$. Um fato importante é que a coluna de C-18 não sofre modificação aparente e pode ser reutilizada diversas vezes seguindo o mesmo procedimento de limpeza e condicionamento. Durante todos os experimentos não foi observada variação do desempenho da coluna de C-18.

\section{RESULTADOS E DISCUSSÃO}

\section{Estudo sobre a composição da solução impregnante}

A solução para coletar seletivamente o $\mathrm{NO}_{2}$ usada inicialmente para condicionar a coluna de $\mathrm{C}$ - 18 foi a proposta por VINGIMOORI e LING $^{18}$ com pequena modificação. A solução aquosa contendo $22 \%$ (v/v) de TEA, 3,6\% (v/v) etilenoglicol e 25\%(v/v) de acetona é indicada como solução absorvente para condicionar peneiras moleculares usadas em tubos de amostragens para $\mathrm{NO}_{2}$ atmosférico. Usou-se a mesma solução para condicionar a coluna de C-18. A capacidade da coluna de C-18 preparada com esta composição de solução foi avaliada para coletar o analito presente em um padrão ga-

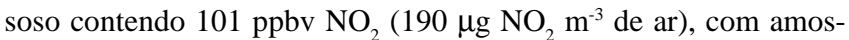
tragem feita durante 60 min e vazão de amostragem de $0,5 \mathrm{~L} \mathrm{~min}^{-1}$. Foram repetidos nove experimentos nas mesmas condições, e o sinal analítico resultante possui uma absorbância média igual a 0,232 , com coeficiente de variação igual a 3,3\%. Os resultados mostraram uma repetibilidade do sinal o que indica que a coluna de $\mathrm{C}-18$ manteve a capacidade de coleta constante durante os vários experimentos.

Experimento descrito na literatura ${ }^{22}$ sugere que é complexa a interação entre solução absorvente e substrato resultando em uma forte dependência entre a capacidade de coleta e a composição da solução impregnante adicionada por massa de substrato. Tendo em vista este fato, procurou-se testar diferentes composições da solução 
impregnante buscando aumentar a capacidade de coleta da coluna de C-18 utilizada na amostragem. Os resultados de experimentos feitos com colunas de $\mathrm{C}-18$ preparadas com diferentes composições da solução impregnante, mas avaliadas nas mesmas condições e com a mesma composição do padrão gasoso estão listados na Tabela 1. Os resultados indicam que a solução impregnante contendo a metade da quantidade de TEA inicialmente usada, apresentou uma eficiência 57\% maior no sinal analítico, mostrando uma melhora na capacidade de coleta pela superfície. $O$ resultado sugere que a quantidade de TEA distribuída sobre a superfície que forma o leito impregnado influencia diretamente na capacidade de coleta do $\mathrm{NO}_{2}$. Possivelmente a eficiência de coleta do $\mathrm{NO}_{2}$ depende da interação do $\mathrm{NO}_{2}$ com ambos: suporte sólido e a TEA, e o excesso de TEA forma um filme espesso sobre o suporte sólido, diminuindo a possível interação necessária para a coleta eficiente do $\mathrm{NO}_{2}$.

Tabela 1. Testes de eficiência de coleta para a coluna C-18 preparada com diferentes composições da solução impregnante. Amostra de ar contendo 101 ppbv de $\mathrm{NO}_{2}$, vazão de amostragem de $0,5 \mathrm{~L} \mathrm{~min}^{-1}$, tempo de amostragem de $60 \mathrm{~min}$

\begin{tabular}{lcc}
\hline \multicolumn{2}{c}{ Composição solução(\% v/v) } & Absorbância, $540 \mathrm{~nm}$ \\
TEA & ETILENOGLICOL & \\
\hline 22,0 & 3,6 & 0,230 \\
11,0 & 3,6 & 0,365 \\
44,0 & 3,6 & 0,187 \\
22,0 & 1,8 & 0,228 \\
22,0 & 7,2 & 0,237 \\
22,0 & 0,0 & 0,235 \\
\hline
\end{tabular}

Efeito da vazão de amostragem sobre a capacidade de coleta do $\mathrm{NO}_{2}$

A vazão de amostragem é um parâmetro necessário de se avaliar, pois muitos sistemas de amostragem são limitados pela vazão e funcionam em uma estreita faixa de trabalho. A coluna C-18 foi avaliada para a faixa de vazão de amostragem entre 0,5 e $1,7 \mathrm{~L} \mathrm{~min}^{-1} \mathrm{com}$ padrões gasosos contendo $30 \mathrm{ppbv}$ de $\mathrm{NO}_{2}$, e um tempo de amostragem de $1 \mathrm{~h}$. Os resultados dos experimentos estão representados na Figura 2. Como indica a figura, dentro da faixa de vazão avaliada o sinal analítico expresso em absorbância (A) aumenta linearmente com o aumento da vazão de amostragem (Q) segundo a equação:

$A=16,46 \cdot 10^{-2} \mathrm{Q}+2,10 \cdot 10^{-2}(\mathrm{R}=0,9743)$

Aumentando-se a vazão de amostragem de uma mistura gasosa contendo uma mesma concentração de $\mathrm{NO}_{2}$ significa que um maior número de moléculas estará chegando ao amostrador em um mesmo período de tempo. A dependência diretamente linear entre sinal analítico e vazão de amostragem, nestes experimentos, indicam que a coluna de $\mathrm{C}$-18 usada na amostragem retêm proporcionalmente a maior quantidade de $\mathrm{NO}_{2}$ que chega ao amostrador quando se aumenta a vazão de amostragem. Portanto, dentro da faixa de vazão estudada a coluna de $\mathrm{C}-18$ coleta $\mathrm{NO}_{2}$ em quantidades proporcionais à sua presença em amostras de ar.

\section{Efeito do tempo de amostragem sobre a capacidade de coleta da coluna C-18}

O tempo de amostragem é um parâmetro que deve ser muito bem dimensionado no desenvolvimento do método analítico envol-

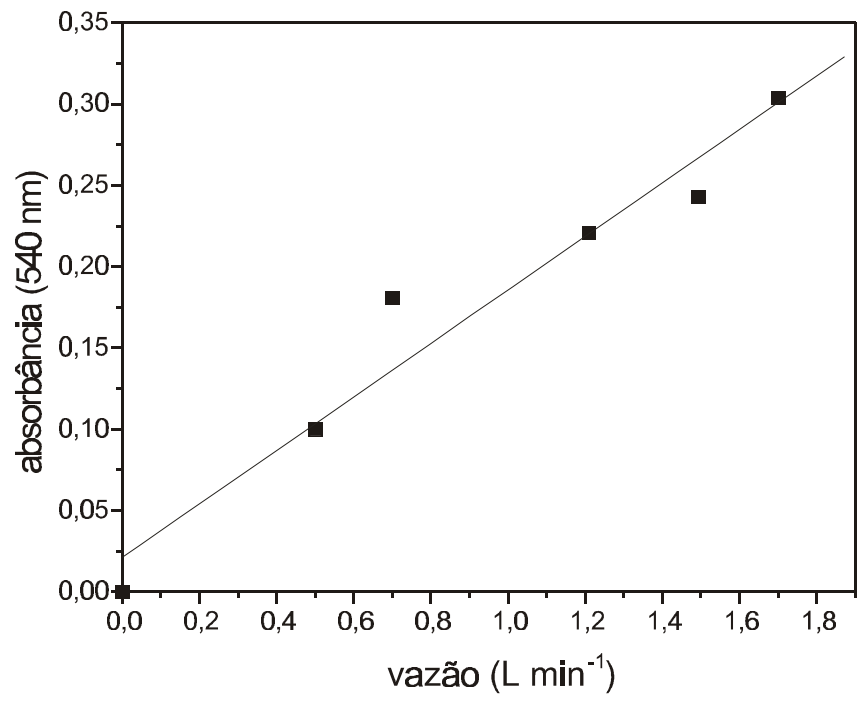

Figura 2. Efeito da vazão na capacidade de coleta de $\mathrm{NO}_{2}$ pelo tubo de amostragem. Concentração $30 \mathrm{ppbv}$ de $\mathrm{NO}_{2}$, tempo de amostragem de 60 $\min$

vendo etapa de preconcentração. Tempos curtos podem resultar em uma quantidade de analito insuficiente para ser quantificada, enquanto que tempos de amostragem muito longos podem extrapolar a capacidade de coleta do amostrador resultando em perda do analito na fase de amostragem e conseqüentemente resultando em um dado analítico que não representa a amostra. Para se estipular o limite do tempo de amostragem, deve-se conhecer qual o tempo em que o amostrador pode funcionar sem que ocorra a saturação do leito sorvente e conseqüentemente perdas do analito. Para o estudo da influência do tempo na amostragem foi usada uma vazão de amostragem de 1,5 $\mathrm{L} \mathrm{min}^{-1}$ e um padrão gasoso contendo 34 ppbv de $\mathrm{NO}_{2}$. Para avaliar a possibilidade de saturação da coluna de amostragem, utilizou-se duas colunas de C-18 conectadas em série. A função da segunda coluna de C-18 foi identificar possível saturação da capacidade de coletar parte do $\mathrm{NO}_{2}$ que chega para ser amostrado na primeira coluna. Como se pode observar pelos resultados da Figura 3, a primeira coluna de C-18 mantém sua eficiência de

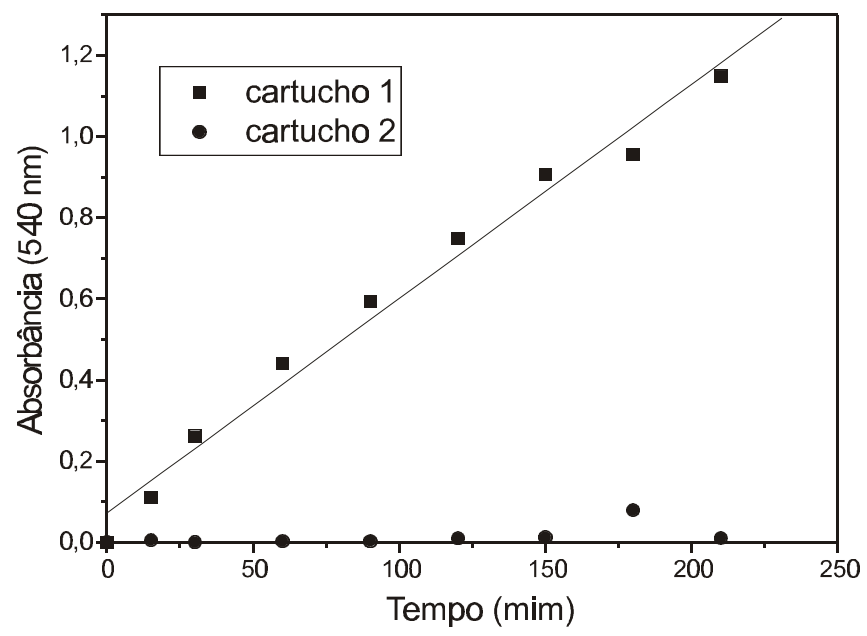

Figura 3. Avaliação da capacidade de coleta de $\mathrm{NO}_{2}$ pelo cartucho de amostragem. Concentração $34 \mathrm{ppbv}$ de $\mathrm{NO}_{2}$. Vazão amostragem de $1,5 \mathrm{~L}$ min ${ }^{-1}$. Tempo de amostragem: 15 - 210 mim. Cartuchos 1 e 2: colunas $C$ - 18 impregnadas com TEA e conectadas em série na linha de amostragem 
coleta dentro do tempo avaliado (10 e $210 \mathrm{~min}$ ), apresentando um aumento linear de absorbância (A) proporcional ao aumento do tempo de amostragem (t, min), segundo a equação:

$A=5,3 \cdot 10^{-3} t+72,4 \cdot 10^{-3}(R=0,9914)$

Uma saturação da primeira coluna de C-18 deveria resultar em um desvio da linearidade com a quantidade do analito tendendo para um valor constante. A segunda coluna deveria também reter o $\mathrm{NO}_{2}$ não coletado pela primeira coluna. No tempo de amostragem avaliado a solução resultante da amostragem na segunda coluna não apresentou sinal de absorbância significativo, fato que comprova a não saturação do primeira coluna. Não se estudou tempo superior a 210 min, por ser muito superior ao tempo previsto para amostragens utilizando este método.

\section{Curva de calibração e comparação de métodos}

Após o reconhecimento dos principais parâmetros que governam a retenção do $\mathrm{NO}_{2}$ pela coluna de C-18 impregnada com a solução de TEA, passamos a avaliar a sua capacidade de resposta para diferentes concentrações de $\mathrm{NO}_{2}$. A amostragem foi feita a $0,5 \mathrm{~L} \mathrm{~min}^{-1}$ durante 60 min. Como resultado foi construída uma curva de calibração, onde a absorbância (A) medida foi linearmente proporcional à concentração de $\mathrm{NO}_{2}$ (ppbv) presente nos padrões gasosos (Figura 4). Este comportamento pode ser expresso pela equação abaixo:

$\mathrm{A}=(42,5 \cdot \pm 0,7) \cdot 10^{-4}\left[\mathrm{NO}_{2}\right]+(15,9 \pm 29,4) \cdot 10^{-4}(\mathrm{R}=0,9988)$

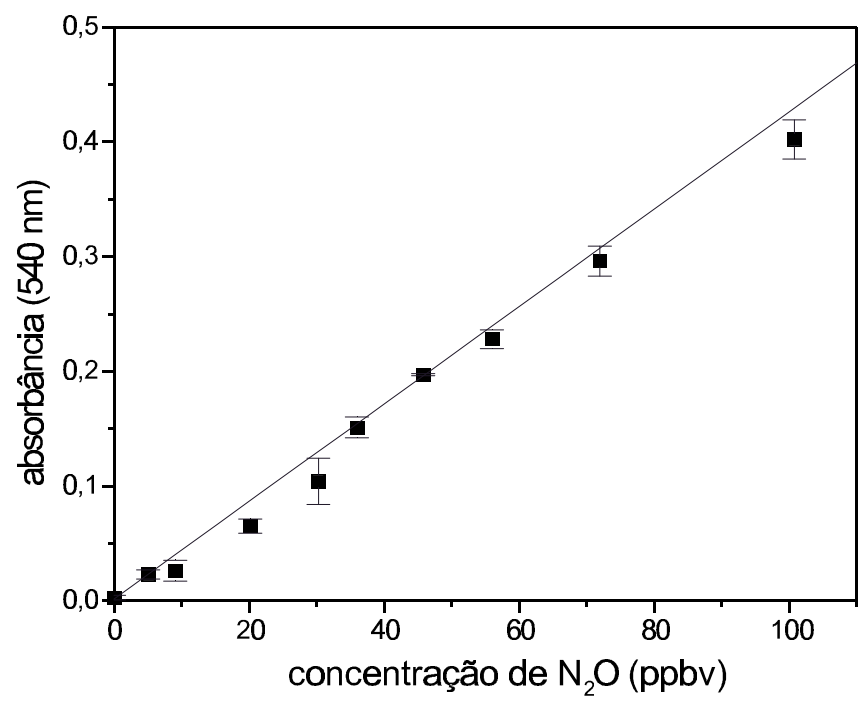

Figura 4. Curva de calibração para faixa de concentração de $\mathrm{NO}_{2}$ entre 10 e 100 ppb. Vazão: 0,5 L min ${ }^{-1}$. Tempo de amostragem: $60 \mathrm{~min}$

Não se trabalhou com concentrações menores que 5 ppbv devido a dificuldade de se gerar padrões gasosos contendo quantidades pequenas de $\mathrm{NO}_{2}$. Para gerar padrões gasosos contendo quantidades muito pequenas de $\mathrm{NO}_{2}$ são necessários grandes volumes de gás diluente. Grandes vazões de gás em laboratório são difíceis de serem medidas e apresentam erro elevado. Uma alternativa seria gerar padrões gasosos de $\mathrm{NO}_{2}$ dentro de um recipiente de volume fixo, o que evita o erro de diluiçã̃o, porém por outro lado, aumenta o erro relacionado com o fenômeno de adsorsão do $\mathrm{NO}_{2}$ muito diluído nas paredes do recipiente. Este dilema no preparo de padrões gasosos de baixa concentração foi discutido por Taggat e colaboradores ${ }^{24}$. O limite de detecção calculado para o método proposto neste trabalho foi de 1,4 ppbv, que é suficiente para a aplicação do método na determinação de $\mathrm{NO}_{2}$ tanto em ambientes abertos como fechados. Porém, com amostragens mais longas e/ou vazões maiores, limites inferiores certamente podem ser alcançados, .

Como o método aqui descrito foi proposto como uma forma alternativa ao método proposto por Saltzman ${ }^{11}$, o qual usa borbulhadores para coletar $\mathrm{NO}_{2}$, seria conveniente comparar os resultados obtidos para uma mesma amostra de ar. Com este objetivo, foi montado um conjunto de borbulhadores contendo solução de GriessSaltzman seguindo o procedimento descrito em manual sobre análise de gases ${ }^{12}$. Amostragens de ar foram feitas em paralelo usando os dois métodos e após o preparo conveniente das soluções foram feitas as leituras de absorbância. Os resultados de absorbância obtidos em amostragens feitas em borbulhadores $(0,174 \pm 0,013)$ foram $52 \%$ menores em relação aos resultados obtidos com o método proposto neste trabalho $(\mathrm{A}=0,365 \pm 0,015)$. Isto mostra que o método aqui descrito permite coletar e determinar o $\mathrm{NO}_{2}$ com eficiência, além de ter a vantagem de ser mais prático do que borbulhadores de vidro para amostragens em campo.

\section{Estudo de interferentes}

A ação dos interferentes na determinação do $\mathrm{NO}_{2}$ já foi muito estudada e se restringe à etapa de reação de Griess-Saltzman com relação a possíveis espécies interferentes ${ }^{11}$. Com exceção ao $\mathrm{NO}_{2}$, os demais óxidos de nitrogênio são inativos. $\mathrm{O}$ nitrato de peroxiacetila (PAN), resultante da reação de compostos orgânicos voláteis (COV) e $\mathrm{NO}_{x}$ via reações fotoquímicas ${ }^{25}$, pode produzir até $35 \%$ do seu equivalente molar como $\mathrm{NO}_{2}$, mas como sua concentração na atmosfera é muito pequena comparada com o $\mathrm{NO}_{2}$, sua interferência é desprezível. Oxidantes como o ozônio em contato com o reagente de Griess-Saltzman, podem causar interferência que tem seu máximo efeito $3 \mathrm{~h}$ depois de iniciada a reação. Fazendo-se a leitura colorimétrica até $1 \mathrm{~h}$ após iniciada a reação de formação do azo composto esta interferência pode ser minimizada. O gás dióxido de enxofre $\left(\mathrm{SO}_{2}\right)$ é um interferente potencial dos métodos de análise de $\mathrm{NO}_{2}$ na etapa de amostragem possivelmente pela reação que ocorre entre estes dois compostos. Assim procuramos dar neste estudo especial atenção a este tipo de interferência. Para verificar a interferência deste composto foram preparados padrões gasosos contendo quantidades conhecidas de $\mathrm{NO}_{2}$ e $\mathrm{SO}_{2}$. Como fonte de $\mathrm{SO}_{2}$ foi usado um tubo de permeação com taxa média de permeação de $917 \mathrm{ng} \mathrm{min}^{-1} \pm$ $11 \%$ a $30{ }^{\circ} \mathrm{C}$. Mesmo quando a concentração de $\mathrm{SO}_{2}$ foi aproximadamente 7 vezes maior $\left(\left[\mathrm{NO}_{2}\right]=101 \mathrm{ppbv}\right.$ e $\left.\left[\mathrm{SO}_{2}\right]=712 \mathrm{ppbv}\right)$ não se observou interferência significativa já que o valor da absorbância medida $\left(\mathrm{A}\left(\mathrm{NO}_{2} / \mathrm{SO}_{2}\right)=0,352\right)$ foi apenas cerca de $5 \%$ menor que os valores obtidos para padrões gasosos contendo apenas $\mathrm{NO}_{2}$ na mesma concentração $\left(\mathrm{A}\left(\mathrm{NO}_{2}\right)=0,372\right)$,o que sugere que o método é eficiente mesmo na presença de quantidades significativamente maiores de $\mathrm{SO}_{2}$ como é o caso de quando queimam combustíveis fósseis com alto teor de enxofre. Possivelmente a composição da solução de TEA usada tem influência na minimização da interferência pelo $\mathrm{SO}_{2}$. Saltzman ${ }^{11}$ sugere a adição de acetona para minimizar a ação do $\mathrm{SO}_{2}$ como interferente, neste caso possivelmente o uso da acetona na composição da solução de TEA utilizada no preparo do leito sorvente atua minimizando a interferência do $\mathrm{SO}_{2}$.

\section{Aplicação do método para determinação de $\mathrm{NO}_{2}$ em amostras de ar atmosférico}

O método proposto foi utilizado para se determinar a variação de $\mathrm{NO}_{2}$ durante alguns dias do outono de 2000 na cidade de 
Araraquara (SP). Estas medidas tiveram como objetivo apenas aplicar o método à amostras do ambiente e portanto sem qualquer pretensão de um estudo ambiental amplo. As amostras foram coletadas no Instituto de Química (UNESP) que está localizado no limite oeste do perímetro urbano. A cidade é de médio porte com cerca de 180 000 habitantes e está localizada distante de grandes centros urbanos. Cada amostragem foi feita com 60 min de duração e com vazão de $0,5 \mathrm{~L} \mathrm{~min}^{-1}$. Os resultados obtidos mostraram que a concentração de $\mathrm{NO}_{2}$ varia entre cerca de 4 até $50 \mathrm{ppb}$, ao longo do dia. Quando é comparado estes resultados com experimento semelhante feito na cidade de Claremomnt, Califórnia, USA cerca de $50 \mathrm{Km}$ a leste do centro de Los Angeles ${ }^{26}$ observa se que os perfis de variações são bastante semelhantes, mas com valores menores para cidade de Araraquara, como era de se esperar para uma cidade de médio porte e sem grandes fontes significativas de $\mathrm{NO}_{2}$.

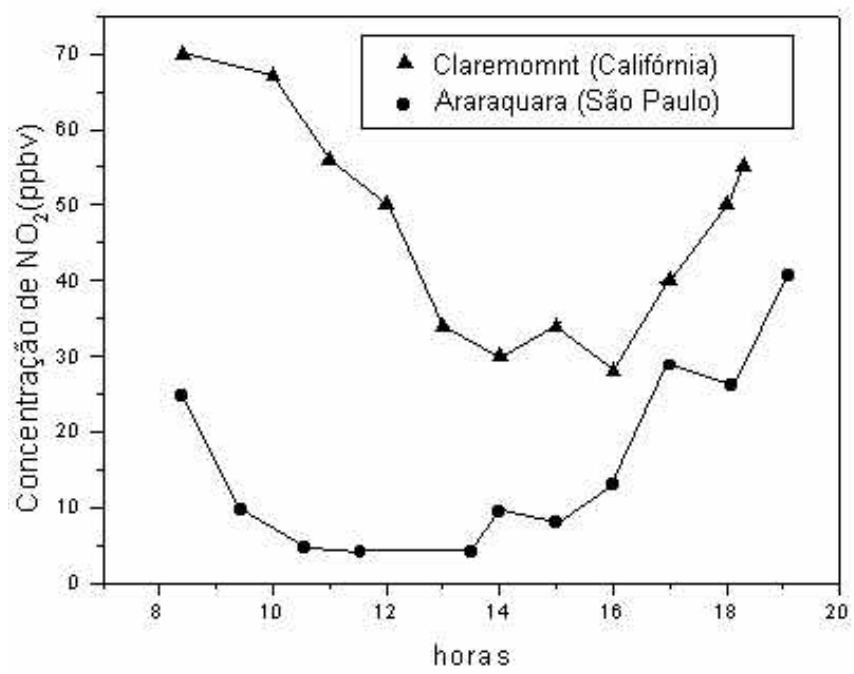

Figura 5. Variação da concentração de $\mathrm{NO}_{2}$ ao longo do dia (outono de 2000) na cidade de Araraquara (São Paulo - Brasil) e comparação com os valores de concentração obtidos em Claremomnt (Califórnia - USA $)^{26}$

O método proposto apresentou em análises de campo um comportamento similar ao obtido no laboratório com padrões gasosos. As colunas de C-18 apresentaram uma facilidade maior de uso quando comparado com borbulhadores de vidro e apresentaram melhores resultados de coleta. O método é sensível o suficiente para medir possíveis variações de concentração de $\mathrm{NO}_{2}$ na atmosfera ao longo do dia tanto em ambiente aberto como fechado.

\section{AGRADECIMENTOS}

Os autores agradecem o suporte financeiro concedido pelo CNPq e C. Ugucione agradece à FAPESP pela bolsa concedida.

\section{REFERÊNCIAS}

1. Logan, J.A.; J. Geophys. Res. 1983, 88, 10785.

2. Singh, H.B.; Environ. Sci. Technol. 1987, 21, 320.

3. Lobert ,J.M.; Scharffe, D.H.; Hao, W.M.; Crutzen, P.J.; Nature 1990, 34,552 .

4. Manahan, S.E. Em Environmental Chemistry; Lewis Publishers,1994, p. 338.

5. WHO/UNEP (World Health Organization/ United Nations Environment Programme) Environmental Health Criteria 188, Nitrogen Oxides; World Health Organization, 1997, p. 74.

6. Gatward, J. ; Colls, J. J.; Environ. Technol. 1990, 11, 381.

7. Noy, D.; Brunekreef, B. ; Boleij, J. S. M.; Houthuijs, D.; De Koning, R.; Atmos. Environ. 1990, 24 A, 1521

8. Ugucione, C.; Cardoso, A. A.; Gomes Neto, J. A.; Resumos da 24a Reunião Anual da Sociedade Brasileira de Química, Poços de Caldas, Brasil,2001.

9. Goldstein, I. F.; Andrew, R. L.; Hartel D.; Atmos. Environ. 1988, 22, 2127.

10. Zakrzewsk, S.P.; Principles of Environmental Toxicology; American Chemical Society; 1997, p. 133.

11. Saltzman, B. E.; Anal. Chem. 1954, 26, 1949.

12. Levaggi, D. A.; Appel, B. R.; Horstman, D. W.; Kothny, E. L.; Wendt, J. G.; Em Methodos of Air Sampling and Analysis; Lodge Jr, J. P., ed.; Lewis: Chelsea, 1989, p. 389.

13. Van Mourik, J. H. C.; Am. Ind. Hyg. Assoc. J. 1965, 26, 498.

14. Levaggi, D. A.; Siu, W.; Feldstein, M.; Kothny, E. L.; Environ. Sci. Technol. 1972, 6, 250 .

15. Cuddeback, J. E.; Saltzman, B. E.; Burg, W. R.; J. Air Pollut. Control Assoc. 1975, 25, 725.

16. Adams, K. M.; Japar S. M.; Pierson, W. R.; Atmos. Environ. 1986, 20, 1211.

17. Levaggi, D.A. ;Sui, W.; Feldstein, M.; J. Air Pollut. Control Assoc. 1973, 23,30 .

18. Vinjamoori, D.V.; Ling, C.S.; Anal.Chem. 1981, 53, 1689.

19. Sickles II, J. E.; Grohse, P. M.; Hodson, L.L.; Salmons, C. A.; Cox, K. W.; Turner, R.; Estes, E. D.; Anal.Chem. 1990, 62, 338.

20. Krochmal, D.; Gorski, L.; Environ. Sci. Technol. 1991, 25, 531.

21. Wang, Y.; Allen, A. G.; Mark, D.; Harrison, R. M.; J. Environ. Monit. 1999, 1,423 .

22. Rodrigues, B. A.; Pitombo, L. R. M.; Cardoso, A. A.; J. Braz. Chem. Soc. 2000, 11, 71.

23. Krochmal, D. ; Kalina, A.; Atmos. Environ. 1997, 31, 3473.

24. Mac Taggart, D. L.; Kagel, R. A.; Farwell, S.; J. Air Pollut. Control Assoc. 1987, 37, 143.

25. Suppan, P. P.; Fabian, P.; Vyras, L.; Gryning, S. E.; Atmos. Environ. 1998, 32, 2089.

26. Apud Finlayson-Pitts, B. J.; Pitts Jr.; Atmospheric Chemistry: Fundamentals and Experimentals Techniques; John Wiley \& Sons: New York, 1986, p. 571. 